Draft version November 17, 2018

Preprint typeset using $\mathrm{IAT}_{\mathrm{E}} \mathrm{X}$ style emulateapj v. 5/2/11

\title{
PLANETESIMAL INTERACTIONS CAN EXPLAIN THE MYSTERIOUS PERIOD RATIOS OF SMALL NEAR-RESONANT PLANETS
}

\author{
Sourav ChatTerJee \\ Center for Interdisciplinary Exploration \& Research in Astrophysics (CIERA) \\ Physics \& Astronomy, Northwestern University, Evanston, IL 60202, USA \\ sourav.chatterjee@northwestern.edu and \\ Department of Astronomy, University of Florida, Gainesville, FL 32611, USA. \\ ERIC B. FORD \\ Department of Astronomy \& Astrophysics \\ The Pennsylvania State University, 525 Davey Laboratory, University Park, PA 16802, USA and \\ Center for Exoplanets and Habitable Worlds \\ The Pennsylvania State University, 525 Davey Laboratory, University Park, PA, 16802, USA \\ eford@psu.edu \\ Draft version November 17, 2018
}

\begin{abstract}
An intriguing trend among Kepler's multi-planet systems is an overabundance of planet pairs with period ratios just wide of a mean motion resonance (MMR) and a dearth of systems just narrow of them. Traditional planet formation models are at odds with these observations. They are also in contrast with the period ratios of radial-velocity-discovered multi-planet systems which tend to pile up at 2:1 MMR. We propose that gas-disk migration traps planets in a MMR. After gas dispersal, orbits of these trapped planets are altered through interaction with a residual planetesimal disk. We study the effects of planetesimal disk interactions on planet pairs trapped in 2:1 MMR using planets of mass typical of the Kepler planet candidates (KPC) and explore large ranges for the mass, and density profile of the planetesimal disk. We find that planet-planetesimal disk interactions naturally create the observed asymmetry in period-ratio distribution for large ranges of planetesimal disk and planet properties. If the planetesimal disk mass is above a threshold of $\approx 0.2 \times$ the planet mass, these interactions typically disrupt MMR. Afterwards, the planets migrate in such a way that the final period-ratio is slightly higher than the integer ratio corresponding to the initial MMR. Below this threshold these interactions typically cannot disrupt the resonance and the period ratio stays close to the integer ratio. The threshold explains why the more massive planet pairs found by RV surveys are still in resonance. We encourage future research to explore how significantly the associated accretion would change the planets' atmospheric and surface properties.

Subject headings: scattering-methods: N-body simulations-methods: numerical-planetary systemsplanetary systems: protoplanetary disks-planetary systems: formation-planets and satellites: general
\end{abstract}

\section{INTRODUCTION}

NASA's Kepler mission has revolutionized our understanding of planetary systems, their multiplicity, and occurrence rate (Borucki et al. 2010, 2011, Batalha et al. 2013 Burke et al. 2014: Rowe et al. 2014 Fabrycky et al. 2014). Most of the candidates have radii between $\sim R_{\oplus}$ to Neptune radius $\left(R_{\text {Neptune }}\right)$. Several trends, not all apriori expected, have emerged among this new class of small (presumably low-mass) planet population. One of the most prominent trends is the existence of extremely compact, well aligned, short period multi-transiting systems (e.g., Fang \& Margot 2012: Hansen \& Murray 2012 2013 Chiang \& Laughlin 2013 Chatterjee \& Tan 2014). Another trend is that although the spacings between planet pairs among most KPCs seems random, there is a clear overabundance of pairs just wide of major MMRs including 2:1, and 3:2, (Figure 1) and a lack of planet pairs just inside of these resonances (Lissauer et al. 2011. Fabrycky et al. 2014). This feature in the period-ratio distribution in the KPCs is in drastic contrast to that observed in the planet populations discovered via RV sur- veys (Butler et al. 2006) which preferentially find much higher mass planets, and shows a clear peak very near the 2: 1 MMR. Tests for the statistical significance of this apparent overabundance indicate a clear deficit of planet pairs interior to $2: 1$ and an overabundance near period ratio of 2.2 (Steffen \& Hwang 2014).

Smooth gas-disk driven convergent migration is expected to trap planets into MMRs resulting in period ratios that are very close to the exact integer ratios, and this is thought to be responsible for the giant planet MMR pairs (e.g., Lee \& Peale 2002, Armitage 2013). The orbital period ratios of a pair of planets near the $j+1: j$ MMR usually have small $\left(10^{-3}\right)$ offsets from the exact integer ratios, $\epsilon \equiv P_{2} / P_{1}-(j+1) / j \lesssim 10^{-3}$, where $P_{i}$ denotes the orbital period of the $i^{t h}$ planet (e.g., $\epsilon=P_{2} / P_{1}-2 / 1$ for $\left.2: 1 \mathrm{MMR}\right)$. Planet-planet scattering, on the other hand, brings large changes to the orbits of the planet pairs initially trapped into a MMR and likely dramatically increases the relative inclinations of these orbits making multi-transiting configuration impossible (e.g., Rasio \& Ford 1996; Chatterjee et al. 2008 


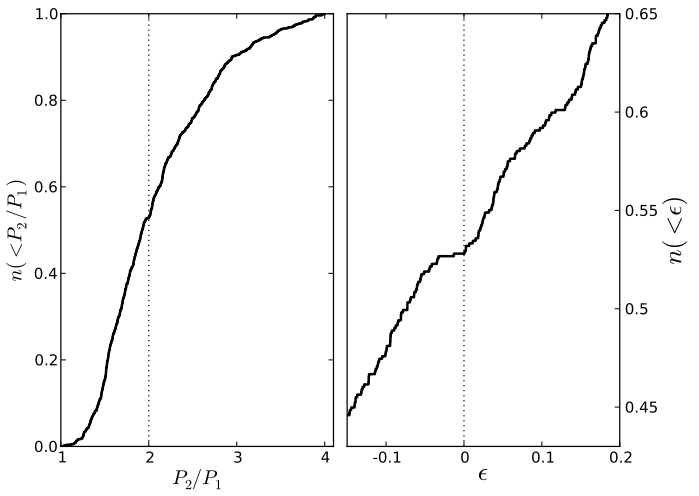

FIG. 1.- Cumulative histogram for the period ratios $\left(P_{2} / P_{1}\right)$ of adjacent planet pairs discovered by Kepler (left). Right panel shows a zoomed in version of the same as a function of $\epsilon \equiv P_{2} / P_{1}-$ 2. The vertical dotted lines denote the exact positions for the $2: 1$ MMR.

Jurić \& Tremaine 2008; Nagasawa \& Ida 2011; Beaugé \& Nesvorny (2012). Hence, the process responsible for the observed large $(\lesssim 0.2)$ positive $\epsilon$ distribution must not be as smooth as gas-disk migration and also not as strong as planet-planet scattering. The mechanism responsible for this feature must be fairly common during planetary system formation, as evidenced by the high fraction of near-resonance KPC pairs exhibiting this feature. This process must also be significantly more powerful for lowmass planets compared to the giants where this feature has not been observed.

Several theories have been proposed to explain this feature, (Lithwick \& Wu 2012, Rein 2012, Batygin \& Morbidelli 2013; Petrovich et al. 2013 Delisle \& Laskar 2014). For example, resonance repulsion in presence of tidal damping (Lithwick \& Wu 2012, Batygin \& Morbidelli 2013) creates positive $\epsilon$, but predicts values that are at least an order of magnitude too small suggesting additional dissipative processes are at play (Lee et al. 2013). In situ growth of planets via planetesimal accretion (Petrovich et al. 2013) assumes idealized and likely unphysical initial conditions since planetesimal accretion naturally results in changes in the semi major axis $(a)$. Gas disk interactions in presence of turbulence may result in positive $\epsilon$, but the results are strongly dependent on the strength of such turbulence which is largely uncertain and highly variable.

This leads us to search for a mechanism that can easily create asymmetric shifts in periods from exact MMRs, that is expected to be ubiquitous within the core-accretion paradigm of planet formation, and that is not overly sensitive on the details of the initial conditions. We propose that gas-disk driven migration traps some planet pairs in MMR with low $\epsilon$, as expected from conventional theories (e.g., Lin et al. 1996, Goldreich \& Tremaine 1980, Bryden et al. 2000). After gas dispersal, these planets interact with planetesimals from a residual disk, expected to be present from the coreaccretion paradigm of planet formation. These interactions are stochastic, but of much weaker strength than planet-planet scattering. Planetesimal driven migration (in presence or absence of a gas disk) is a well studied process and has been long identified as an important in- gredient in understanding the formation and evolution of planets, especially, in the context of the outer Solar system (e.g., Fernandez \& Ip 1984: Hahn \& Malhotra 1999. Levison et al. 2007: Kirsh et al. 2009: Bromley \& Kenyon 2011: Ormel et al. 2012; Minton \& Levison 2014). More recently, numerical results suggest that planetesimal disk scattering can significantly alter $\epsilon$ for some specific $\mathrm{Ke}$ pler systems (Moore et al. 2013).

We systematically study the effects of planetesimal disk interactions on resonant planet pairs. In particular, we focus on the 2:1 MMR in this study since the difference in the period ratio distribution between lowmass planets and giant planets is the most dramatic near this MMR. In \$2 we describe our numerical setup, the explored parameter space, and explain the choices of our initial conditions. In 93 we describe our key results. Finally, we discuss the strengths and weaknesses of this process and explain implications that can be observationally tested in 84

\section{NUMERICAL MODELING}

A fully realistic numerical model of systems containing two planets trapped in a MMR emerging from a full protoplanetary disk with planets, planetesimals, and gas is impractical and beyond the scope of this study. Instead, we use pure $N$-body models with initial conditions generated to mimic the expected properties of a system emerging from a dissipative gas disk. In order to create reasonable initial conditions for the planet and planetesimal orbits we adopt a 3-stage algorithm. In Stage 1 we create orbital properties for planet pairs in 2:1 MMR by migrating the outer planet inwards (\$2.1). In Stage 2 we create initial planetesimal disk properties that are dynamically consistent with the resonant planet pair properties $(\$ 2.2)$. These two steps create initial conditions for the final and main stage of models including two resonant planets and a dynamically active planetesimals disk (Stage 3; 2.3 ). We test whether late stage (after gas disk is dispersed) interactions between the resonant planets and a residual planetesimal disk can create large positive offsets from initial integer ratio of periods expected of the MMR. We use the hybrid integrator of MERCURY6.2 (Chambers 1999) for all stages of our simulations. We use a planetary density of $1.64 \mathrm{~g} \mathrm{~cm}^{-3}$, typical of Kepler's multi-planet systems (e.g., Howe et al. 2014: Weiss \& Marcy 2014, Chatterjee \& Tan 2015), to calculate the planet sizes for all our simulations. The planetesimal density is chosen to be $6 \mathrm{~g} \mathrm{~cm}^{-3}$. Below we describe the detailed numerical treatments for each stage of our simulations.

\subsection{Stage 1: Trapping planet pairs into 2 : 1 mean motion resonance}

In this stage our initial properties consist of two planets of masses $m_{1}$ and $m_{2}$ (indices are counted from inside out). The initial semimajor axis for the inner planet is $a_{1}=0.5 \mathrm{AU}$. Initial semimajor axis of the outer planet $a_{2}$ is chosen to be $0.02 \mathrm{AU}$ outside the exact period ratio of $P_{2} / P_{1}=2$. Initially both orbits are circular. Orbital planes of the two planets are aligned initially. We choose other orbital phase angles uniformly from their full ranges. The two planets are evolved using the hybrid integrator. We mimic gas disk damping and convergent migration by applying a forced migration using 


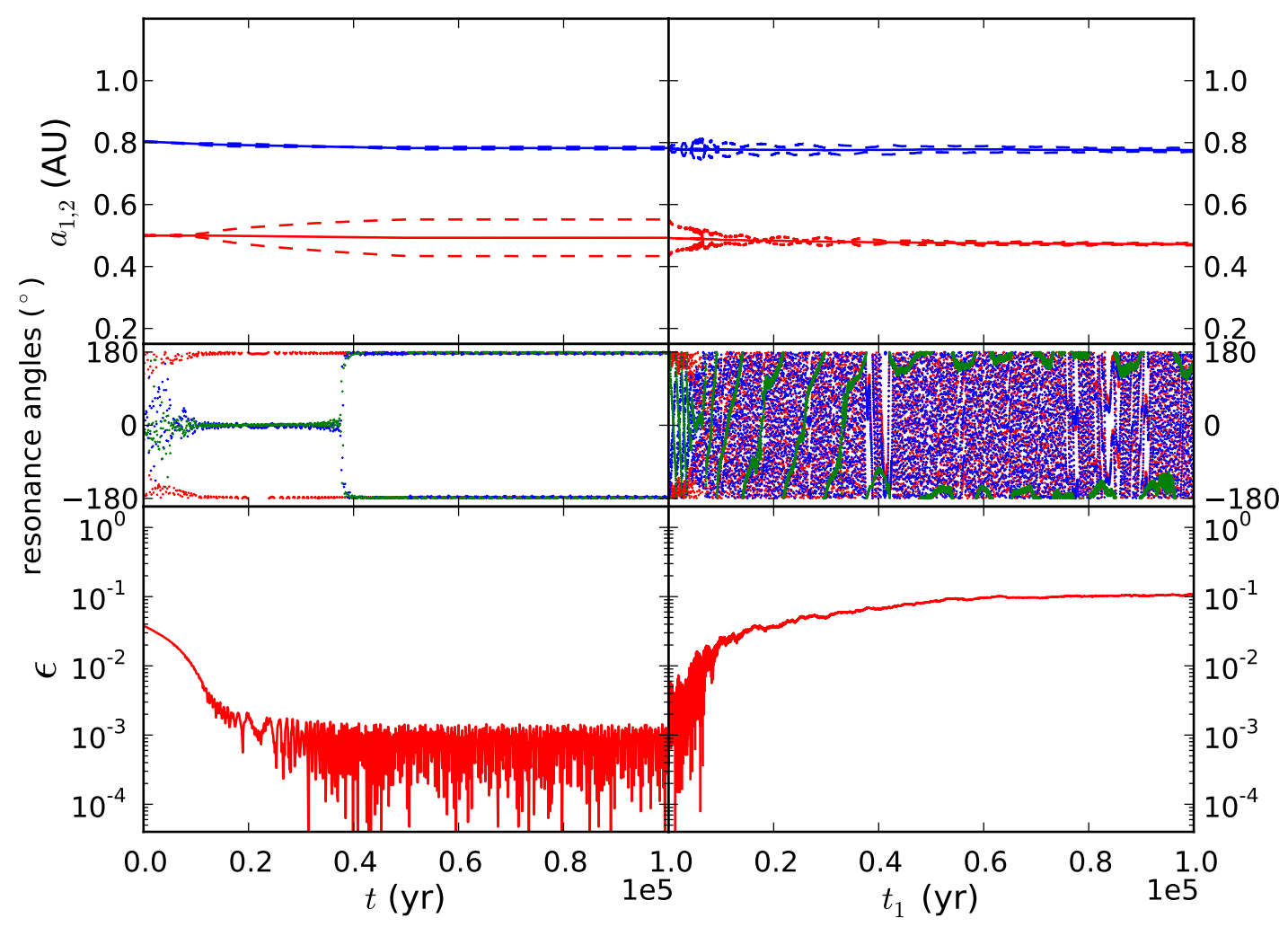

FIG. 2.- Example orbital evolution of resonant planet pairs. For this example we choose one realization with initial properties $m_{1} / m_{2}=1$, $m_{d} / m_{p}=0.5$, and $\alpha=-1.5$. Top panels show the evolution of the semimajor axes (solid), pericenter and apocenter distances (dashed). The middle panels show the evolution of three resonant angles for $2: 1$ resonance. The bottom panels show the evolution of $\epsilon=P_{2} / P_{1}-2 / 1$. Left panels: The evolution of planet pairs during Stage 1 (2.1). The resonant angles are initially circulating indicating non-resonance. These angles start librating when convergent migration traps the two orbits in 2:1 MMR. Orbital eccentricities increase due to resonance trapping. The offset $\epsilon$ steadily decreases until it reaches $\epsilon \sim 10^{-3}$. Right panels: The evolution of the same resonant planets in presence of a planetesimal disk. Planets interact with the planetesimals. Orbital eccentricities are damped. Initially, $\epsilon$ changes chaotically due to individual planet-planetesimal interactions. After several such interactions the overall perturbations disrupt the resonance indicated by recirculation of the resonant angles, in this example, at $t_{1} \sim 5 \times 10^{3} \mathrm{yr}$. Once the resonance is disrupted, $\epsilon$ approaches $\approx 0.1$.

$\dot{a_{2}}=10^{-6} \mathrm{AUyr}^{-1}$ and $\dot{e_{2}}=10^{-4} \mathrm{yr}^{-1}$ on the outer planet (Lee \& Peale 2002). The outer planet moves in smoothly and the two orbits get trapped into 2:1 MMR at $t \approx 2 \times 10^{4} \mathrm{yr}$. The trapped planets move further inwards together for another $2 \times 10^{-4} \mathrm{yr}$, when the $\dot{a_{2}}$ and $\dot{e_{2}}$ terms are switched off. We follow the evolution of the resonant planets till $t=10^{5} \mathrm{yr}$. The left panels in Figure 2 show an example of the evolution of the planetary orbital properties in Stage 1. Initially the resonant angles circulate and the planets are not in resonance. Resonant angles start liberating as the planets are trapped into 2:1 MMR. The value of $\epsilon$ steadily decreases and reaches a small value $\epsilon \lesssim 10^{-3}$. At the end of Stage 1 we note the orbital properties of the planet pair and thus initial orbital properties for a resonant planet pair are created.

\subsection{Stage 2: Creation of dynamically consistent planetesimal disk}

We first choose a disk described by a simple power-law of the form $d \Sigma / d a=a^{\alpha}$. We ascertain that the disk edges are sufficiently far from the planets to avoid unpredictable edge effects by setting the inner disk edge at $0.01 \mathrm{AU}$ interior to the $a$ value for 1: 3 period ratio with the inner planet. Similarly, we set the outer edge of the disk at $0.01 \mathrm{AU}$ exterior to the $a$ value for the $3: 1$ period ratio with the outer planet orbit. The planetesimal positions are selected consistent with the power-law profile. The planetesimal eccentricities $(e)$ are drawn uniformly in the range 0 to $10^{-3}$. The initial orbital inclinations are drawn randomly to vary between $-1^{\circ}$ and $1^{\circ}$. Other orbital phase angles for the planetesimals are chosen randomly in their full ranges.

In a real system, while a gas disk is present, it can damp dynamical excitations in the planetesimal orbits caused by the resonant planets. However, planetesimal orbits that are unstable on very short timescales will still be disrupted via collisions and scattering due to strong gravitational interactions with the planets (e.g., Matsumura et al. 2010). As the system emerges out of the gas disk, the planetesimal disk will not remain a pure power-law, but will have features such as a decrease in density near the planets that perturb the initial powerlaw, resonance features, and a larger dispersion in $e$.

We model the planetary perturbations to the planetesimal disk surface density profiles in the following way. We collect final orbital properties of the resonant planet pairs created in Stage 1. We evolve these planets with a swath of planetesimals with properties obtained using 


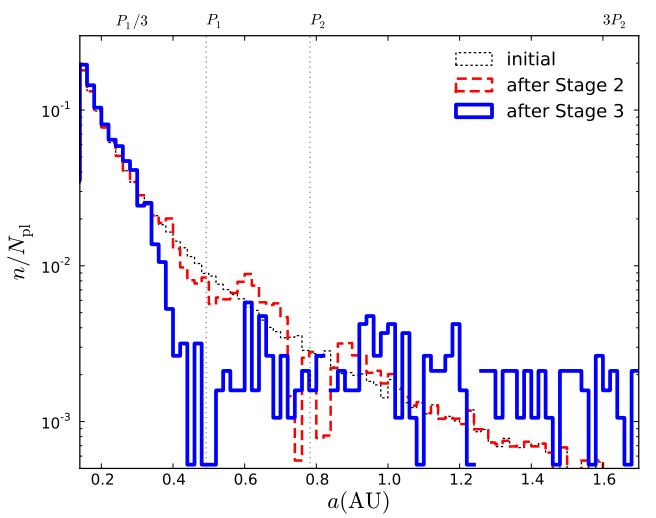

FIG. 3. - Example evolution of the planetesimal disk profile for the same model as in Figure 2 Dotted (black), dashed (red), and thick solid (blue) histograms show the initial undisturbed powerlaw profile, the profile for all planetesimals that survived until the end of Stage 2, and the final profile for planetesimals at the end of Stage 3, respectively. The vertical dotted lines show the positions of the planets at the beginning of Stage 3 . The positions of $P_{1} / 3$ and $3 P_{2}$ are shown as well, for reference since these values are used to determine the positions of the disk edges $(2.2$. Each histogram is normalized by the total number of planetesimals at each stage $\left(10^{5}, 99653\right.$, and 1892 for initial, after Stage 2, and after Stage 3 , respectively). The presence of the planet pair alter the powerlaw profile. So the planetesimal surface density exhibits dynamical features at the end of Stage 2. At the end of Stage 3 only a small number of planetesimals remain close to the planets.

a particular value of $\alpha$ and disk edges as described above for $100 \mathrm{yr}$. At this stage we treat all planetesimals as test particles, so the planets can dynamically alter the planetesimal orbits, but the planetesimals cannot change planet properties. After $t=100 \mathrm{yr}$ we stop the integrations and collect orbital properties of the surviving planetesimals. We create a large database of $N \sim 10^{5}$ surviving planetesimal orbits for each $\alpha$ and each combination of $m_{1}$ and $m_{2}$. The database will not include planetesimals on orbits that rapidly become unstable, for example, via physical collisions with the planets. However, if planetesimals can survive in specific orbital configurations, such as 1: 1 resonance, horse-shoe or tadpole orbits, then such orbits will be naturally populated among the orbital database of surviving planetesimals. Hence, at the end of Stage 2, a database of planetesimal orbits is created that is dynamically consistent for each combination of resonant planet pair mass and initial planetesimal disk surface density profile (e.g., Figure 3).

We expect that shortly before gas disk dispersal $e$ damping may not be efficient and eccentricities of planetesimal orbits near the planets will likely grow (Matsumura et al. 2010). Therefore, during Stage 2 we do not apply any forced damping. As a result, planetesimal eccentricities grow freely via encounters with the planets. The exact values depend on the details of the initial planet and planetesimal disk properties. We find that the median $e$ for all planetesimal orbits at the end of Stage 2 is $\sim(6.1 \pm 0.5) \times 10^{-3}$. For planetesimal orbits within $a_{1}-10 R_{H, 1}$ and $a_{2}+10 R_{H, 2}\left(R_{H, i}\right.$ is the Hill radius of the $i^{\text {th }}$ planet), a region within which almost all planetesimals will have a strong encounter with the planets during Stage 3, the median $e$ is a little higher $\sim(1.5 \pm 0.2) \times 10^{-2}$. Here the error bars denote the standard deviation in the $e$-distributions.
Our simulations do not explicitly include a gas disk. Rather, they are designed to imitate the expected effects of a gas disk, namely, removal and alterations of only those orbits that are unstable on short timescales ( $\sim 100 \mathrm{yr}$ is our choice), and preservation of orbits that are not unstable on these short timescales. The integration stopping time of $100 \mathrm{yr}$ in Stage 2 is somewhat arbitrary since it is hard to predict the stabilizing effect of the gas disk and exactly which planetesimals will be unstable even while the gas is present. Our choice of $100 \mathrm{yr}$ results in removing planetesimal orbits unless they are stable for $\gtrsim 3 \times 10^{2}$ orbits of the inner planets. Hence, further interactions at orbital timescales are not expected. Integrating longer would not change the overall qualitative results as long as there are enough planetesimals in the disk to interact with the planets at the end of this stage.

\subsection{Stage 3: Evolution of initially resonant planet pairs in presence of a massive planetesimal disk}

This is the main stage of our study where simulations follow the evolution of planet pairs in resonance embedded in a dynamically consistent planetesimal disk. We randomly choose $N_{\mathrm{pl}}=2 \times 10^{3}$ planetesimal orbits from the orbital databases we create in Stage 2 for each combination of a resonant planet pair and initial power-law exponent $\alpha$. We extract the orbital properties for the resonant planet pair at the end of Stage 2. We evolve the planets and planetesimals together. Now, the planetesimals are treated as pseudo-test particles, such that planetesimals interact with the planets, but they do not interact between themselves. This approximation reduces the computational cost and is not expected to change our results significantly. All planetesimals are of equal mass in each simulated model. The mass of each planetesimal is set to achieve the desired ratio between the total planetesimal disk mass $\left(m_{d}\right)$ and the total planet mass $m_{p}=m_{1}+m_{2}$. We stop our integrations at $t_{1}=10^{5} \mathrm{yr}$. We have tested that longer integrations and integrations with larger $N_{\mathrm{pl}}$ (at fixed $m_{d}$ ) do not alter the results in a statistically significant way. At the end of the integrations we retrieve the orbital properties of the planets and all planetesimals. We study the evolution of orbital properties of the initially resonant planets including $\epsilon$ and the resonant angles.

\subsection{Exploration of Parameter Space}

We restrict our study to the 2:1 MMR since $\epsilon$-values for Kepler systems show the strongest difference from RV-discovered systems near period ratio of 2 , primarily due to a large number of RV-discovered giant planet pairs near a period ratio of 2 .

We choose the planet masses in the following way. The more massive planet's mass is set to $5 \times 10^{-5} M_{\odot} \approx$ $1 M_{\text {Neptune. }}$ The mass of the other planet, is determined using a grid of $m_{1} / m_{2}$ values. We vary $m_{1} / m_{2}=$ $0.1,0.2,1,5$, and 10. For example, for $m_{1} / m_{2}=5$, $m_{1}=5 \times 10^{-5} M_{\odot}, m_{2}=10^{-5} M_{\odot}$ and for $m_{1} / m_{2}=0.2$, $m_{1}=10^{-5} M_{\odot}, m_{2}=5 \times 10^{-5} M_{\odot}$.

We vary the disk density profile given by $d \Sigma / d a \propto a^{\alpha}$ by choosing $\alpha$ in a large range between -2.5 to 3 using a grid step-size of 0.5 . For each combination of $m_{1}, m_{2}$, and $\alpha$ we vary $m_{d} / m_{p}$ between 0.1 to 1 with a grid step- 
size of 0.1 . In addition, we include $m_{d} / m_{p}=1.5$. To address the inherent statistical fluctuations we simulate 4 realizations for each choice of $m_{1} / m_{2}, \alpha$, and $m_{d} / m_{p}$. Thus, our results are based on a large ensemble $(2620$ simulations in Stage 3, each with two planets trapped in 2: $1 \mathrm{MMR}$ and $2 \times 10^{3}$ pseudo-test particles) of models.

\subsection{Number of planetesimals}

The choice of $N_{\mathrm{pl}}$ in Stage 3 is somewhat arbitrary and guided by the following considerations. In this study we will test whether the cumulative effect of many small perturbations from several planetesimals can eventually disrupt the 2:1 resonance between a pair of planetary orbits, and leave them close to the initial period commensurability with $\epsilon \sim 0.01$ to 0.2 . Hence, it is essential to ensure that the perturbation caused by an individual planet-planetesimal interaction is sufficiently small and cannot disrupt the resonance. For a given $m_{d} / m_{p}$ the higher the $N_{\mathrm{pl}}$, the smoother the evolution. However, the computational cost scales as $\sim N_{\mathrm{pl}}$ for the pseudo test-particles. Combined with the requirement of small timesteps (we use 1 day) to increase accuracy and a sufficiently long integration time, making $N_{\mathrm{pl}}$ very large is computationally impractical. Fortunately, analytical considerations from resonance theory can guide us to determine a sufficient value of $N_{\mathrm{pl}}$ while keeping the computational costs reasonable.

We require that the maximum possible fractional change $\delta a / a$ to a planet's orbit caused by dynamical interaction with a single planetesimal is lower than the resonance width $\Delta a / a$ of the MMR. The libration width $|\Delta a / a|$ for 2: $1 \mathrm{MMR}$ in the restricted three-body case is

$$
\left|\frac{\Delta a}{a}\right| \geq 78.43 \frac{m_{p}}{m_{\star}}
$$

where $m_{p}$ and $m_{\star}$ are the planet mass and the star mass, respectively (Murray \& Dermott 1999). The maximum change in $\delta a / a$ from a single planet-planetesimal interaction is

$$
\left|\frac{\delta a}{a}\right|_{\mathrm{pl}} \approx 2 \frac{m_{\mathrm{pl}}}{m_{\star}}
$$

where $m_{\mathrm{pl}}$ is the mass of the planetesimal. Hence, our requirement is satisfied if

$$
\frac{m_{\mathrm{pl}}}{m_{p}} \leq 39.22 \frac{m_{p}}{m_{\star}} .
$$

Using our model assumptions of $m_{\star}=1 M_{\odot}, m_{p}=5 \times$ $10^{-5} M_{\odot}$, and for equal mass planetesimals, the above condition is equivalent to $N_{\mathrm{pl}} \gtrsim 5 \times 10^{2}$. Numerical tests of Stage 3 simulations using varying $N_{\mathrm{pl}}$ verify that any $N_{\mathrm{pl}} \gtrsim 5 \times 10^{2}$ gives statistically indistinguishable results, and $N_{\mathrm{pl}} \gtrsim 10^{3}$ makes the evolution fairly smooth. Hence, the choice of $N_{\mathrm{pl}}=2 \times 10^{3}$ in our models is adequately high for this study. Using $N_{\mathrm{pl}}=2 \times 10^{3}$ and by treating them as pseudo-test particles, simulations in Stage 3 took a total of $\sim 5 \times 10^{5}$ CPU hours to complete.

\section{RESULTS}

Scattering and accretion of the planetesimals perturb the planetary orbits stochastically (see Figure 2, Right Panels for an example). Initially, $\epsilon$ changes chaotically

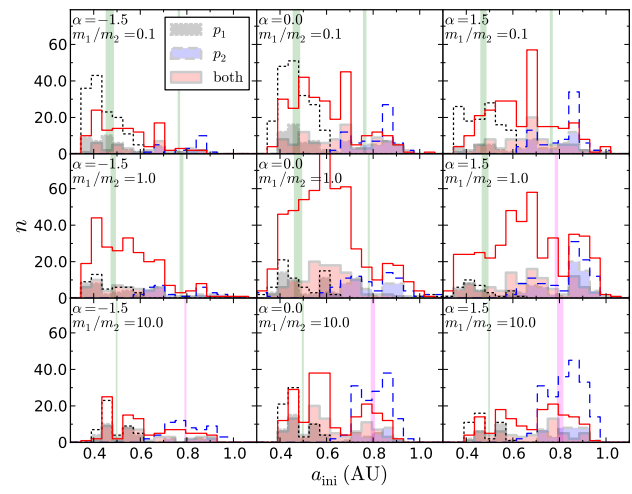

FIG. 4.- Distributions for the initial positions of planetesimals that came within $1 R_{H}$ of only planet 1 (black dashed), only planet 2 (blue long-dashed), and both planets (red solid) during Stage 3 evolution. Filled histograms denote the same, but includes only planetesimals that collided with one of the planets in the end. Each panel shows a particular combination of initial $m_{1} / m_{2}$ and $\alpha$ (noted in each panel). All panels are for $m_{d} / m_{p}=0.5$. The vertical shaded regions are bounded by the initial and final planet positions. Green and red for the shaded regions denote inward and outward migration, respectively. In all of these example cases the inner planet migrated inward. When the outer planet also migrates inward, it does so at a lower rate compared to the inner planet resulting in growth of $\epsilon$ in all of these cases.

due to individual planet-planetesimal interactions. No single interaction is strong enough to break the resonance. These interactions damp the eccentricities of both planets. After sufficiently large number of interactions the overall perturbations disrupt the resonance indicated by recirculation of the resonance angles. Once the resonance is disrupted, further interactions steadily increase $\epsilon$ until there are insufficient planetesimals left in the disk that can interact with the planets (e.g., Figure 3). Then $\epsilon$ reaches a steady value, $\sim 0.1$ in this example (Figure 2 .

For a representative set of our models $\left(m_{d} / m_{p}=0.5\right.$, $\alpha=-1.5,0,1.5$, and $\left.m_{1} / m_{2}=0.1,1,10\right)$ we turn on extensive logging to track close encounters, defined as planetesimals coming within 1 Hill radius $\left(R_{H}\right)$ of a planet. Outcomes of each planet-planetesimal close encounter depends on the details of the initial conditions including $m_{1} / m_{2}$ and $\alpha$ (Figure 4). For our choice of planet properties (mass and density), planetesimals from a large range in initial positions $\left(0.3 \lesssim a_{\text {ini }} / \mathrm{AU} \lesssim 1\right)$ come within $1 R_{H}$ of both planets. Planetesimals that come within $1 R_{H}$ of either the inner or the outer planet had $a_{\text {ini }}$ predominantly inside of the inner planet or outside of the outer planet. The fraction of all planetesimal orbits at the beginning of Stage 3 that come within $1 R_{H}$ of a planet varies widely depending on $m_{1} / m_{2}$ and $\alpha$. For example, $\approx 6 \%$ of all planetesimals come within $1 R_{H}$ of a planet during the Stage 3 evolution for one model with $m_{1} / m_{2}=1, \alpha=1.5, m_{d} / m_{p}=0.5$, whereas, this fraction is $\approx 50 \%$ for another model with $m_{1} / m_{2}=0.1$, $\alpha=-1.5$ and $m_{d} / m_{p}=0.5$. The fraction of planetesimals taking part in close encounters also vary by large fractions from model to model with the same initial $m_{1} / m_{2}, \alpha$, and $m_{d} / m_{p}$ but different simply by random realization of the initial conditions of planetesimals orbits (e.g., $5 \%$ and $12 \%$ between two models with $m_{1} / m_{2}=1$, $\alpha=1.5$, and $\left.m_{d} / m_{p}=0.5\right)$. The ratio of the number 


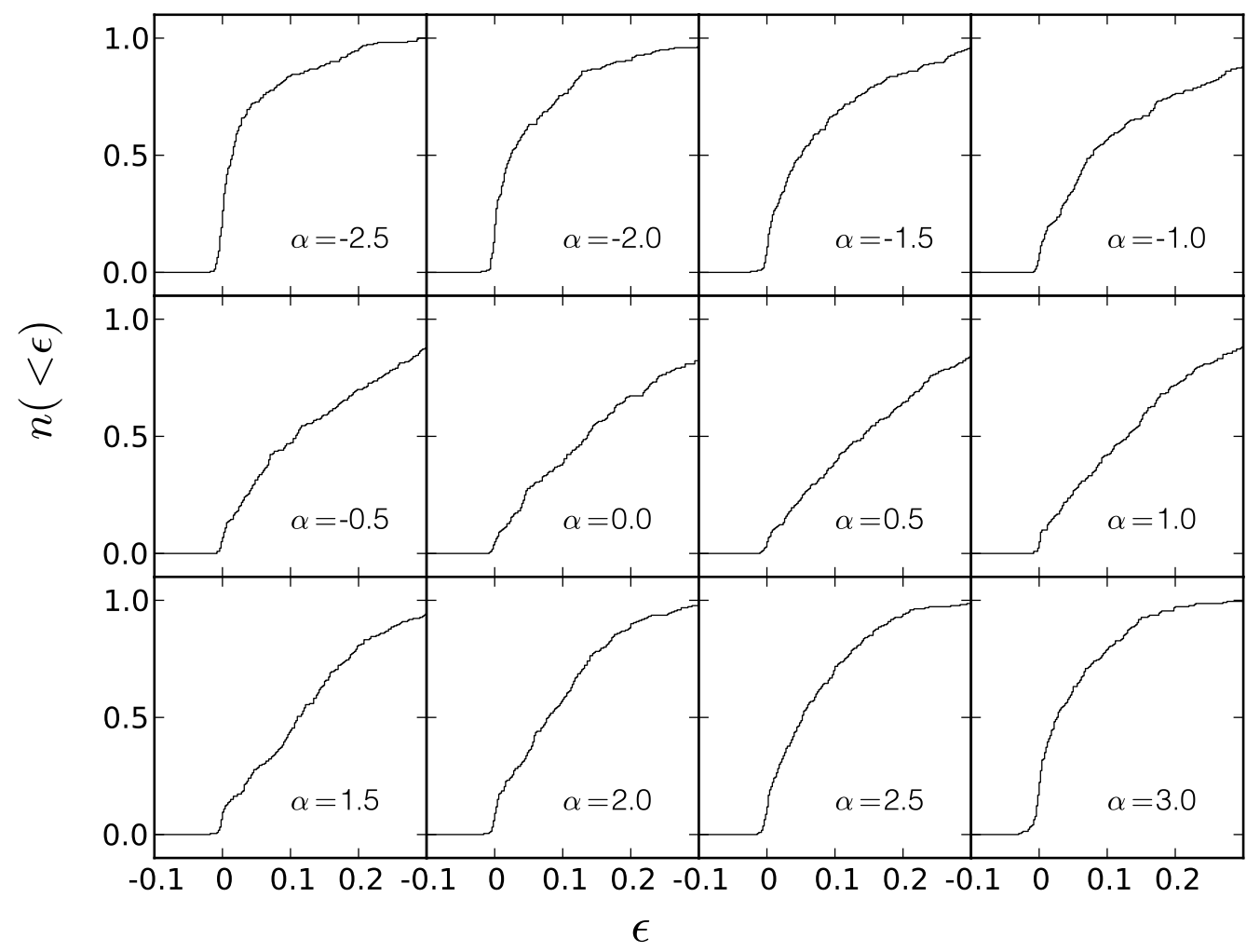

Fig. 5.- Cumulative histogram of the final $\epsilon$ as a result of the interactions between a planetesimal disk and resonant planet pairs. Each panel shows results from models with all $m_{1} / m_{2}$ and $m_{d} / m_{p}$ but with a particular $\alpha$, denoted in the panels. Planetesimal interactions predominantly create $\epsilon>0$ and naturally explains the asymmetry in the period ratio distribution observed in the Kepler data, regardless of the local disk profile.

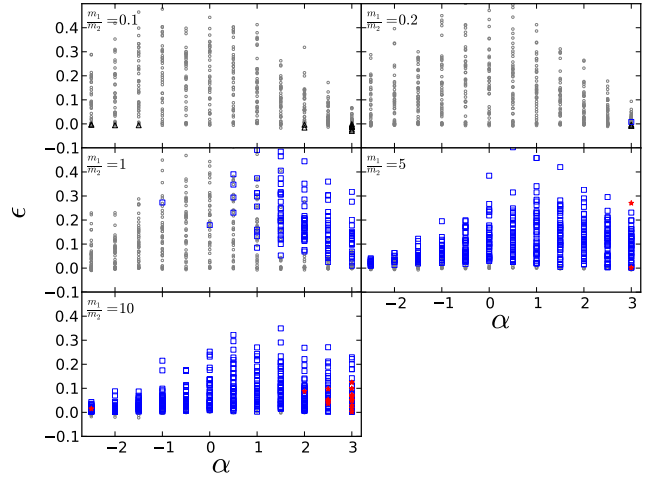

FIG. 6. - $\epsilon$ vs $\alpha$ for all studied initial $m_{1} / m_{2}$, each panel for a given value noted in the corresponding panel. Grey dots, black triangles, blue squares, and red stars denote cases where both $a_{1}$ and $a_{2}$ decreased, $a_{1}$ increased and $a_{2}$ decreased, $a_{1}$ decreased and $a_{2}$ increased, and both $a_{1}$ and $a_{2}$ increased after Stage 3 , respectively. Depending on the details of initial properties such as $m_{1} / m_{2}$ and $\alpha$ the direction of migration varies for the individual planets. However, for sufficiently massive planetesimal disks, $\epsilon$ typically grows to significant positive values.

of planetesimals that has at least one close encounter with the planets and the number of planetesimals that physically collide with one of the planets also can vary widely $\left(\approx 20 \%\right.$ for one of our models with $m_{1} / m_{2}=1$, $\alpha=-1.5, m_{d} / m_{p}=0.5$, and $40 \%$ for $m_{1} / m_{2}=10$, $\left.\alpha=-1.5, m_{d} / m_{p}=0.5\right)$. The fraction of accreted planetesimals that had close encounters with the planets prior to accretion varies stochastically between realizations with the same initial values for $m_{1} / m_{2}, \alpha$, and $m_{d} / m_{p}$. For example, two different models both with $m_{1} / m_{2}=1, \alpha=-1.5$ and $m_{d} / m_{p}=0.5$ show $24 \%$ and $33 \%$ for this quantity. Planetesimals that finally collide with one of the planets, often had multiple close encounters with both planets prior to accretion. Among models with extensive logging of this information we find that the ratio of the number of accreted planetesimals that had multiple close encounters with both planets before they were finally accreted and the number of all accreted planetesimals is typically large and varies between $41 \%$ (for a model with $m_{1} / m_{2}=0.1, \alpha=-1.5$, $m_{d} / m_{p}=0.5$ ) to $65 \%$ (for a model with $m_{1} / m_{2}=1$, $\left.\alpha=-1.5, m_{d} / m_{p}=0.5\right)$.

The quantitative results including when the resonance is broken and the final value of $\epsilon$ vary from model to model. However, the qualitative nature of the evolution for the planetary orbits remains the same for all $m_{1} / m_{2}$, $\alpha$, and $m_{d} / m_{p}$ we have considered. For all models with a sufficiently massive initial planetesimal disk $\left(m_{d} / m_{p} \geqslant\right.$ 0.2 ) the resonance is typically broken and $\epsilon$ grows to positive values for all $\alpha$ (Figure 5). These results indicate that the observed asymmetry in the $\epsilon$-distribution for near-resonance planet pairs in the Kepler data, namely, 


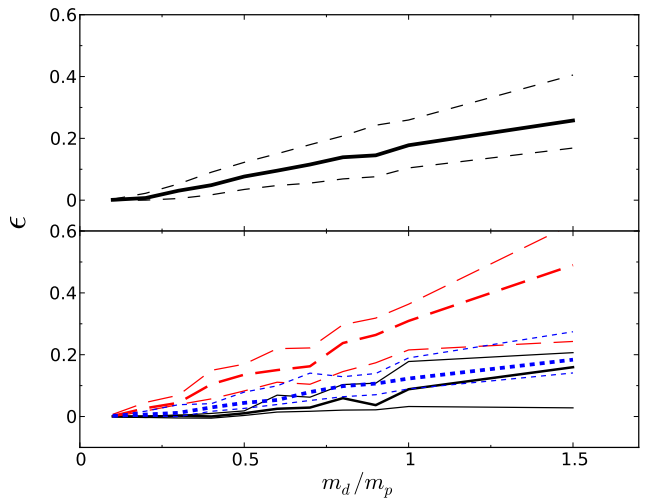

FIG. 7.- Quartile values for the $\epsilon$-distributions as a function of $m_{d} / m_{p}$. The top panel shows results from all our models. The bottom panel shows results from models with three specific planetesimals disk profiles $\alpha=-2.5$ (black solid), 0 (red long-dashed), and 2.5 (blue short-dashed). In each panel, the thick and thin lines show the median, and the $25 \%$ (below) and $75 \%$ (above) percentiles. The quartiles of the $\epsilon$ distributions increase almost linearly with $m_{d} / m_{p}$. The slope and the minimum value for disruption of resonance and growth of $\epsilon$ depends on $\alpha$.

an overabundance of planet pairs just wide of the $2: 1$ and $3: 2$ MMRs and a dearth of systems narrow of them, can be a natural outcome of interactions between resonant planet pairs and planetesimals from a planetesimal disk. This basic outcome is insensitive to the details of the values for $\alpha, m_{1} / m_{2}$, and $m_{d} / m_{p}$ as long as $m_{d} / m_{p}$ is sufficiently high.

For a given $m_{d} / m_{p}$, disks with flatter profiles contain a larger number of planetesimals close to the planetary orbits compared to steeper profiles because of our numerical setup that ensures that the disk edges are always sufficiently far from the planetary orbits $(\$ 2.2)$. As a result, the shape of the final $\epsilon$ distribution depends on $\alpha$ (Figure 5), primarily because $\alpha$ affects the number of planetesimals that can interact with the planets. A flatter disk profile $(|\alpha|<1)$ tends to create larger $\epsilon$ values overall. The extreme positive and negative $\alpha$ models result in very similar distributions for the final $\epsilon$ values.

The migration directions for individual planets do depend on the details of these initial properties (Figure 6). We first focus on systems where the resonance is broken and the final $\epsilon$ has grown significantly $(\gtrsim 0.01)$. Except for a few cases with $m_{1} / m_{2}>1$ and $\alpha>2$ the inner planet always migrates inwards. For $m_{1} / m_{2}<1$, for all $\alpha$, the outer planet also moves inwards but at a smaller rate compared to the inner planet such that the orbits diverge and $\epsilon$ values grow. In contrast, for $m_{1} / m_{2}>1$ the outer planet migrates outwards for all $\alpha$. For $m_{1} / m_{2}=1$, both outcomes are possible depending on $\alpha$. If $\alpha>1$, then planetary orbits diverge via inner planet moving in and outer planet moving out. Otherwise both planets move in. For the cases where $\epsilon$ stays small (e.g., when the resonance is not broken as a result of the planet-planetesimal interactions), $\epsilon$ can have both positive and negative values and both planets may move slightly in or out. We also find that for any given $\alpha$ the dispersion in the growth of $\epsilon$ is typically higher if $m_{1} / m_{2}<1$.

Figure 7 shows the dependence of the final $\epsilon$ on $m_{d} / m_{p}$. The quartiles $(25 \%, 50 \%$, and $75 \%)$ for the final $\epsilon$ distri-

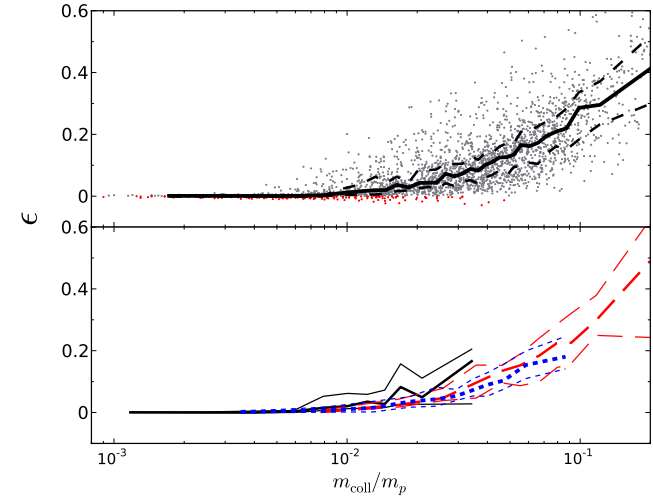

FIG. 8.- Same as Figure 7 but here we show quartile values for the $\epsilon$ distributions as a function of $m_{\text {coll }} / m_{p}$. The grey and red dots in the top panel show the final $\epsilon>0$ and $\epsilon \geq 0$ values, respectively. The quartiles of the $\epsilon$-distributions increase almost linearly both with $m_{\text {coll }} / m_{p}$, similar to $m_{d} / m_{p}$. Clear threshold value for $m_{\text {coll }} / m_{p} \sim 0.008$ can be seen for disruption of resonance and growth of $\epsilon$. The rate of increase of the quartiles as a function of $m_{\text {coll }} / m_{p}$ does not show a strong dependence on $\alpha$.

butions increase almost linearly with increasing $m_{d} / m_{p}$ (Top-Left). For $m_{d} / m_{p} \leqslant 0.2$, the resonance is typically not disrupted and $\epsilon$ remains small $\left(\sim 10^{-3}\right)$. The critical value for $m_{d} / m_{p}$ to break the $2: 1$ resonance and drive up $\epsilon$ depends on $\alpha$ because the number of planetesimals close enough to interact with the planets depends on $\alpha$. For example, for models with $\alpha=-2.5$ all quartiles for the $\epsilon$-distribution remains small for $m_{d} / m_{p} \lesssim 0.5$. The slope of the increase of the quartiles with respect to $m_{d} / m_{p}$ also stays relatively low. For another steep disk profile $\alpha=2.5$ the results are quite similar, and show only a small difference in the exact value of the critical $m_{d} / m_{p}$ for resonance disruption and a modest increase of the slope. In contrast, for a flat disk profile $\alpha=0$ we find that the critical $m_{d} / m_{p}$ value is reduced significantly and the quartiles of the $\epsilon$ distribution show a much steeper slope. These differences largely originate from the difference in the number of planetesimals close enough to interact with the planet pairs depending on $\alpha$. Indeed Figure 4 shows that flat $(\alpha=0)$ disk profiles result in many more planet-planetesimal close encounters compared to steeper profiles (i.e., $\alpha=1.5,-1.5$ ). For the steeper disk profiles a large number of planetesimals do not suffer any strong interactions with the planets simply because they are too far from the planets. Thus, the critical value for $m_{d} / m_{p}$ remains dependent, on the initial power law profile and the locations of the disk edges relative to the planetary orbits.

While $m_{d} / m_{p}$ provides important information for this process such as how much total angular momentum or energy must be stored in the planetesimal orbits (for a given choice of planetesimal boundaries and $\alpha$ ), the quantitative results such as how many planetesimals take part in strong interaction with the planets depend on the choices of the disk profile and the disk edges. For example, if the planetesimal disk spans a smaller region, same degree of planet-planetesimal interactions may be achieved from a lower $m_{d} / m_{p}$. A less ambiguous and more dynamically informative quantity is the total mass of planetesimals that had strong interactions with the 
planets $\left(m_{\text {int }}\right)$

As a proxy to strong interactions we calculate the total mass of planetesimals accreted by the planets $\left(m_{\text {coll }}\right)$. Using the representative models with extensive logging of all close encounters (Figure 4 ) we find that $m_{\text {coll }}$ (equivalently number of collisions) is highly correlated with $m_{\text {int }}$. The Pearson's correlation coefficient between $m_{\text {coll }}$ and $m_{\text {int }}$ is 0.95 . This confirms that $m_{\text {coll }}$ should serve as a good tracer of the total mass of dynamically important planetesimals. Alternatively, several other quantities including the total number of planet-planetesimal strong encounters, or total number of planetesimals within regions close to either planets could have been used. However, using $m_{\text {coll }}$ as a tracer of the mass of dynamically important planetesimals gives us two advantages. Tracking $m_{\text {coll }}$ does not require extensive logging of close encounters which slows these simulations significantly. In addition, physical collisions may lead to observable differences in atmospheric properties and may create strange mass-radius relations for these planets, which make $m_{\text {coll }}$ a very interesting quantity.

The quartiles for the final $\epsilon$-distributions increase almost linearly with increasing $m_{\text {coll }} / m_{p}$, similar to what we see in the case of $m_{d} / m_{p}$ (Figure 8). However, we find that the slopes for the quartiles of $\epsilon$ distributions with respect to $m_{\text {coll }} / m_{p}$ do not vary as much with changing $\alpha$ as was seen for $m_{d} / m_{p}$. Several key factors related to the inherent nature of this process are apparent from the dependence of $\epsilon$ on $m_{\text {coll }} / m_{p}$. Each planetplanetesimal interaction results in a small change in $a$, $\delta a<\Delta a$, the resonance width. The qualitative behavior of each random interaction between a planetesimal and one of the planets is similar to a random walk (bottom right panel, Figure 1) with step size $\delta a$. In this picture, higher $m_{\text {coll }} / m_{p}$ means larger number of steps. If $m_{\text {coll }} / m_{p}$ is not sufficiently high, these random steps are unlikely to drive the planets out of resonance. For a sufficiently large $m_{\text {coll }} / m_{p}$, the cumulative effect of several interactions may result in a overall change $>\Delta a$ disrupting the resonance. Afterwards, planets migrate further apart since they are able to interact with more planetesimals near their new positions. The inherent stochastic nature of this process is manifested by the fact that for any given $m_{\text {coll }} / m_{p}, \epsilon$ can attain a range of final values. Even when $m_{\text {coll }} / m_{p}$ is sufficiently high, the resonance may not be disrupted in a small number of systems. Nevertheless, increase in $m_{\text {coll }} / m_{p}$ increases the probability of disruption of resonance and growth of $\epsilon$.

We find that the threshold for resonance disruption and growth of $\epsilon$ is $m_{\text {coll }} / m_{p} \approx 0.008$, for our choice of planet masses and densities which are guided by typical values observed of the Kepler systems (\$2). If the total mass of accreted planetesimals is lower than this critical value, then there is little chance for growth of $\epsilon$. Such threshold-like behavior naturally explains why the positive $\epsilon \sim 0.01-0.2$ are seen only among much smaller, hence less massive Kepler systems. The total mass in planetesimals after gas dispersal is seldom sufficient to disrupt resonances between the more massive RV-discovered planets. Even if giant and small planets, both get trapped in the same MMRs during the gas-disk dominated evolution, planetesimal interactions produce significant $\epsilon>0$ only for the low-mass planets.

Due to the stochastic nature of this process, sometimes

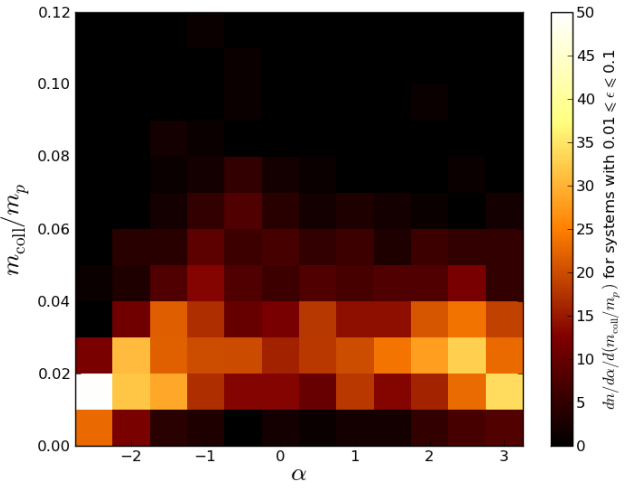

FIG. 9. - Two dimensional histogram of the number of systems with final $0.01 \leq \epsilon \leq 0.1$ as a function of $\alpha$ and $m_{\text {coll }} / m_{p}$. The colors denote the number of systems in each two dimensional bin $d n / d \alpha / d\left(m_{\text {coll }} / m_{p}\right)$. Typically, flatter disks show a larger $m_{\text {coll }} / m_{p}$ range compared to steeper disks for creation of $\epsilon$ values in the same range.

the resonance is not disrupted even if $m_{d} / m_{p}$ or $m_{\text {coll }} / m_{p}$ are sufficiently high. In these cases $\epsilon$ values remain small $\sim 10^{-3}$. Moreover, in such cases, planet pairs can attain both positive and negative $\epsilon$ values. Negative $\epsilon$ values are always small $|\epsilon| \sim 10^{-3}$ (Figure 8) and are only seen in systems where the initial resonance is not disrupted.

In each model we choose a fixed density for the planets. Kepler data, however, show that planets of very similar sizes can have widely varying densities (Marcy et al. 2014; Weiss \& Marcy 2014, Chatterjee \& Tan 2015). Using a few representative models we test whether the extent of divergence of planetary orbits via planetplanetesimal interactions depend on the planetary densities. We find that for the full range in densities (point mass to $0.5 \mathrm{~g} \mathrm{~cm}^{-3}$ ) planetary orbits diverge from the initial resonant configuration and $\epsilon$ can attain large $(\geq 0.01)$ positive values. For example, multiple realizations of models with $m_{1} / m_{2}=1, \alpha=-1.5$, and $m_{d} / m_{p}=0.5$ show final $\epsilon$ values ranging between 0.06 to 0.14 for the point-mass planets, 0.07 to 0.11 for $\rho_{p}=1.68 \mathrm{~g} \mathrm{~cm}^{-3}$, and 0.05 to 0.09 for $\rho_{p}=0.5 \mathrm{~g} \mathrm{~cm}^{-3}$. Hence, we conclude that growth of $\epsilon$ via planetesimal scattering should not critically depend on the choice of planetary densities.

The extent of planetesimal driven migration may depend on the initial $e$ of the planet orbits and the RMS $e$ of planetesimals in Stage $3(\$ 2.2$ 2.3, Kirsh et al. 2009 , Minton \& Levison 2014). Effects of the $e$-distribution in planetesimal orbits on the resonant planets' migration may be interesting to explore in a future study.

Our results indicate that the near-resonant planet pairs in the Kepler data can provide hitherto unprecedented constraints on the structure and mass of the exoplanetesimal disks as the system emerges from the gas disk. For many systems with a pair of near-resonant planets, planet masses can be measured using analysis of transit time variations (e.g., Steffen et al. 2012; Ford et al. 2012; Steffen et al. 2013; Mazeh et al. 2013; Hadden \& Lithwick 2014; Xie 2014). Observational constraints on the planet masses and $\epsilon$ for a particular system can lead to constraints on the total mass and the surface density profile of the planetesimal disk when the gas dissipates, assuming that the $\epsilon$ is generated via planet- 
planetesimal-disk interactions using, e.g., Figure 9 For example, to create the observed $\epsilon=0.18$ for the Kepler$18 \mathrm{bc}$ system, $0.02 \leq m_{\text {coll }} / m_{p} \leq 0.14$ is required for a reasonable range $-\overline{2} .5 \leq \alpha \leq 1$. Disks with steeper density profiles show a smaller range in $m_{\text {coll }} / m_{p}$ to produce the same observed $\epsilon$ (Figure 9) compared to flatter disk profiles.

\section{DISCUSSION}

In this study we propose a mechanism that can naturally change orbits of planet pairs initially trapped in a MMR and create $\epsilon \sim 0.01-0.2$ (Figure 2), typical of the observed KPC pairs near major MMRs (Lissauer et al. 2011; Fabrycky et al. 2014; Steffen \& Hwang 2014). Planetary systems with several planets accompanied by a large number of planetesimals are thought to form naturally via the core accretion paradigm of planet formation (e.g., Goldreich et al. 2004; Armitage 2013). Planetesimal disks are also seen in the Solar system. Planet formation theories also predict trapping of planet pairs via smooth gas-disk driven migration. Hence, our adopted initial setup of resonant planet pairs close to planetesimal disks after gas dispersal is expected to be common based on the predictions of standard planet formation theories. We find that interactions between resonant planet pairs and planetesimals in a planetesimal disk naturally creates large positive $\epsilon$ if the total mass of accreted planetesimals is sufficiently high (Figure 8). These interactions also naturally produce the observed asymmetry in the $\epsilon$ distribution in the Kepler systems for large ranges in $\alpha$ (Figure 5). The existence of a critical value of $m_{\text {coll }} / m_{p}$ (Figure 8) for resonance disruption and growth of $\epsilon$ also explains why this asymmetry is only observed in Kepler systems that are typically less massive compared to the $\mathrm{RV}$-discovered systems.

We conducted pure $N$-body integrations using initial conditions generated to mimic systems that emerged from a dissipative gas disk. To more rigorously ascertain the initial conditions for resonant planet pairs and residual planetesimal disks as they emerge out of a gas disk together, one would need to include gas, planetesimals and planets in a self consistent model. This is extremely challenging and beyond the scope of our study. However, since the basic result of $\epsilon$-growth seems to be common for such a wide range of $\alpha$ (even extreme positive values), these conclusions may not be sensitive to the details of the properties of the planetesimals disk. The only way to avoid our proposed mechanism would be if resonant planet pairs never are near significantly massive planetesimals disks when disk disperses.

If indeed planet-planetesimals interactions are responsible for the observed asymmetric distribution of $\epsilon$ for near resonant planet pairs in the Kepler data, then the high frequency of large- $\epsilon$, near-resonant Kepler systems indicates that planet-planetesimal interactions after gasdisk dissipation is an important evolutionary stage for all small planets independent of their proximity to a resonance. Collisions of planetesimals of total mass $\sim$ few percent of the planet mass is often needed for the observed high values of $\epsilon$ for Kepler-discovered nearresonance planet pairs. Many small Kepler planets are measured to have surprisingly low $\rho_{p}$, which makes collisions even more efficient for these planets. These collisions may affect the planets' atmospheric properties and compositions especially for the low-mass atmospheres believed to be typical for small planets (Rogers 2014). Such accretions could also potentially create an anomalous mass-radius relationship for near-resonant low-mass planets. Therefore, we encourage detailed modeling of the effects of planetesimal collisions on the atmospheric properties of these planets, especially for pairs with large observed $\epsilon$ values.

We thank the referee for his detailed report and constructive comments. This research was supported by NASA Origins of Solar Systems awards NNX09AB35G and NNX13AF61G, NASA Applied Information Systems Research Program award NXX09AM41G, Kepler Participating Scientist Program award NNX12AF73Gm, the University of Florida and the Pennsylvania State University Center for Exoplanets and Habitable Worlds. S.C. also acknowledges support from University of Florida theory postdoctoral fellowship and CIERA fellowship at Northwestern University. The authors acknowledge the University of Florida High-Performance Computing Center for providing computational resources and support that have contributed to the research results reported within this paper.

\section{REFERENCES}

Armitage, P. J. 2013, Astrophysics of Planet Formation

Batalha, N. M., Rowe, J. F., Bryson, et al. 2013, ApJS, 204, 24

Batygin, K. \& Morbidelli, A. 2013, AJ, 145, 1

Beaugé, C. \& Nesvorný, D. 2012, ApJ, 751, 119

Borucki, W. J., Koch, D., Basri, et al. 2010, Science, 327, 977

Borucki, W. J., Koch, D. G., Basri, G., et al. 2011, ApJ, 736, 19

Bromley, B. C. \& Kenyon, S. J. 2011, ApJ, 735, 29

Bryden, G., Różyczka, M., Lin, D. N. C., \& Bodenheimer, P. 2000, ApJ, 540, 1091

Burke, C. J., Bryson, S. T., Mullally, F., et al. 2014, ApJS, 210, 19

Butler, R. P., Wright, J. T., Marcy, G. W., et al. 2006, ApJ, 646, 505

Chambers, J. E. 1999, MNRAS, 304, 793

Chatterjee, S., Ford, E. B., Matsumura, S., \& Rasio, F. A. 2008, ApJ, 686, 580

Chatterjee, S. \& Tan, J. C. 2014, ApJ, 780, 53

Chatterjee, S. \& Tan, J. C. 2015, ApJ, 798, 32

Chiang, E. \& Laughlin, G. 2013, MNRAS, 431, 3444

Delisle, J. B. \& Laskar, J. 2014, A\&A, 570, L7
Fabrycky, D. C., Lissauer, J. J., Ragozzine, D., et al. 2014, ApJ, 790,146

Fang, J. \& Margot, J.-L. 2012, ApJ, 761, 92

Fernandez, J. A. \& Ip, W.-H. 1984, Icarus, 58, 109

Ford, E. B., Ragozzine, D., Rowe, J. F., et al. 2012, ApJ, 756, 185

Goldreich, P., Lithwick, Y., \& Sari, R. 2004, ARA\&A, 42, 549

Goldreich, P. \& Tremaine, S. 1980, ApJ, 241, 425

Hadden, S. \& Lithwick, Y. 2014, ApJ, 787, 80

Hahn, J. M. \& Malhotra, R. 1999, AJ, 117, 3041

Hansen, B. M. S. \& Murray, N. 2012, ApJ, 751, 158

—. 2013, ApJ, 775, 53

Howe, A. R., Burrows, A., \& Verne, W. 2014, ApJ, 787, 173

Jurić, M. \& Tremaine, S. 2008, ApJ, 686, 603

Kirsh, D. R., Duncan, M., Brasser, R., \& Levison, H. F. 2009, Icarus, 199, 197

Lee, M. H., Fabrycky, D., \& Lin, D. N. C. 2013, ApJ, 774, 52

Lee, M. H. \& Peale, S. J. 2002, ApJ, 567, 596

Levison, H. F., Morbidelli, A., Gomes, R., \& Backman, D. 2007, Protostars and Planets V, 669 
Lin, D. N. C., Bodenheimer, P., \& Richardson, D. C. 1996, Nature, 380, 606

Lissauer, J. J., Ragozzine, D., Fabrycky, D. C., et al. 2011, ApJS, 197,8

Lithwick, Y. \& Wu, Y. 2012, ApJ, 756, L11

Marcy, G. W., Isaacson, H., Howard, A. W., et al. 2014, ApJS, 210, 20

Matsumura, S., Thommes, E. W., Chatterjee, S., \& Rasio, F. A. 2010, ApJ, 714, 194

Mazeh, T., Nachmani, G., Holczer, T., et al. 2013, ApJS, 208, 16 Minton, D. A. \& Levison, H. F. 2014, Icarus, 232, 118

Moore, A., Hasan, I., \& Quillen, A. C. 2013, MNRAS, 432, 1196

Murray, C. D. \& Dermott, S. F. 1999, Solar system dynamics

Nagasawa, M. \& Ida, S. 2011, ApJ, 742, 72

Ormel, C. W., Ida, S., \& Tanaka, H. 2012, ApJ, 758, 80
Petrovich, C., Malhotra, R., \& Tremaine, S. 2013, ApJ, 770, 24

Rasio, F. A. \& Ford, E. B. 1996, Science, 274, 954

Rein, H. 2012, MNRAS, 427, L21

Rogers, L. A. 2014, arXiv:1407.4457

Rowe, J. F., Bryson, S. T., Marcy, G. W., et al. 2014, arXiv: 1402.6534

Steffen, J. H., Fabrycky, D. C., Agol, E., et al. 2013, MNRAS, 428,1077

Steffen, J. H., Ford, E. B., Rowe, J. F., et al. 2012, ApJ, 756, 186

Steffen, J. H. \& Hwang, J. A. 2014, arXiv:1409.3320

Weiss, L. M. \& Marcy, G. W. 2014, ApJ, 783, L6

Xie, J.-W. 2014, ApJS, 210, 25 